\title{
彨 University
}

Nashef, L. and Leach, J. P. (2017) SUDEP, the aftermath: supporting the bereaved. Practical Neurology, (doi:10.1136/practneurol-2017-001729).

There may be differences between this version and the published version. You are advised to consult the publisher's version if you wish to cite from it.

http://eprints.gla.ac.uk/149848/

Deposited on: 23 October 2017

Enlighten - Research publications by members of the University of Glasgow http://eprints.gla.ac.uk 
Title: SUDEP, the aftermath: Supporting the bereaved

\author{
Authors: Lina Nashef* and John Paul Leach" \\ *Neurology Department, King's College Hospital, London, UK \\ "School of Medicine, University of Glasgow, UK
}

Key Words: SUDEP, Bereavement, Epilepsy, Aftermath

\title{
Word Count: 2259
}

\section{Summary}

Sudden Unexpected Death in Epilepsy (SUDEP) is a recurring calamity, yet there is little evidence to guide standards of care for supporting the bereaved. Grief in bereavement includes loss, feelings of guilt, anger and blame. In SUDEP, there is also the shock and trauma of the sudden event. How can this be alleviated? While recognising that evidence is lacking and that circumstances may not always facilitate this, this article's focus is on guiding the physician in supporting the bereaved. We propose a pathway of care and mode of communication with the family of the deceased with whom contact is currently limited. We suggest timely contact by telephone or in person, followed by on-going support and referral to voluntary organisations and specialist services, as needed. Clarification and discussion may help mitigate against inappropriate feelings of guilt and blame and may help the family with their sudden and unexpected loss.

\section{SUDEP: The aftermath}

Sudden Death in Epilepsy (SUDEP) is a catastrophic outcome of epilepsy, but inevitable occurrence for anyone in charge of a sizeable clinic. SUDEP is not rare, yet there is little evidence to guide standards of care for supporting the bereaved. Grief in bereavement can include loss, denial, guilt, anger and blame and in SUDEP is compounded by the shock and trauma of the sudden event. What can we do to support families? How could we as physicians deal with our own reaction?

Previously, the UK National Clinical Audit of Epilepsy-Related Death (1) found little documented evidence of contact between clinicians with relatives after death with some $10 \%$ of families contacted by the specialist (adults, $5 \%$; children, $45 \%$ ) and only $7 \%$ by the general practitioner. Such contact was considered particularly important in view of the sudden and unexpected nature of many deaths and the bewilderment, isolation and prolonged distress of families. A recent Australian study showed support provided by professionals was limited following bereavement from epilepsy, even from epilepsy specialists (2). Following in-depth interviews with bereaved relatives (3) many years 
ago in a study of circumstances of death in SUDEP, the same impression favouring early contact was formed. When taken up, such contact is generally valued and can provide much needed support and guidance. It allows bereaved relatives to share recent tragic events and seek information, clarify concerns and clear misunderstandings. NICE guidelines state that 'If a member of your family, or someone you care for, with epilepsy has died unexpectedly, healthcare professionals should contact you so that you can talk about the death. They should also offer to help you contact a SUDEP support group or bereavement counsellors.'(4)

The focus of this article is on guiding the physician in supporting the bereaved. The article proposes a pathway of care while recognising that evidence is lacking and that circumstances may not always facilitate this. The suggestions below are based on the health care system in the United Kingdom which includes general practitioners, specialist epilepsy nurses as well as specialist neurological services. In addition voluntary organisations play an important role. Infrastructure in different countries may vary and the physician's communication and actions would need to be adjusted to the environment and resources where he/she works.

\section{A tragic end which can inform the beginning}

There are no systematic data on the reaction of the treating physician when SUDEP occurs. While dwarfed by the strength of the grief reaction in relatives, we should also acknowledge the grief reaction in health workers when a patient dies unexpectedly "on their watch"(5). The physician's reaction is likely to be complex. Paramount is a deep sadness on hearing of the loss and concern for the bereaved. There is also disappointment in the ultimate failure of medical management. This leads to self-questioning, examination of medical notes, and, potentially, anxiety. Was medical treatment optimal? Was appropriate monitoring, information and support provided to the person with epilepsy and their family? Was documentation adequate? Could anything have prevented the death? Is there likely to be blame? Will there be medicolegal implications?

A number of steps need to be taken. Contacting relatives is important, especially where the family is known to the treating team. If the family have made contact, even if just to inform of the death, we would argue that further communication is mandatory. It may also be necessary to write a medical report for the pathologist or coroner or attend an inquest or Fatal Accident Inquiry. These will require collection of information regarding the death, examining recent events and reviewing medical records, perhaps also involving other professionals including the general practitioner. There may also be an opportunity to consider or facilitate the retention of tissue for future analysis or research according to the family's wishes.

\section{How to contact the bereaved}

Epilepsy specialists vary in the mode of contact. 
a) Letter

Where telephone contact is not possible or appropriate, a letter of condolences is sent. One of us (JPL) prefers to contact the family by letter. Clinicians may feel anxious about such letters, and worry that they will 'say the wrong thing' or make things worse. In practice, no matter how much time has passed since the event, a short letter, sent soon after the clinician learns of the death, expressing sadness and condolences will always be appropriate and readily accepted by a grieving family. This should include an offer of further discussion either in person or by telephone. Experience shows that many families will not accept the offer of discussion, but neither of us is aware of an adverse reaction to this initial approach. Clinicians may copy the letter to the general practitioner. Information can be provided at the same time about self-help groups (SUDEP Action, formerly Epilepsy Bereaved) and bereavement counselling, as appropriate.

\section{b) Telephone Contact}

Some teams prefer to contact the family first by telephone. This may appear more daunting, but where relatives are well-known to the treating team, and if they have made telephone contact, a timely early phone call may well be the best first step. The epilepsy nurse is often first in touch depending on the previous level of contact. Subsequent longer discussions should be pre-arranged, with plenty of time set aside for an unhurried open-ended conversation. Interruptions can impact negatively on the quality of the interaction, and it is important to avoid these. It is appropriate to offer sympathy and condolences, listen to and document the relatives' account of recent events, answer any questions that may arise, and provide guidance where needed. Whether the telephone contact is the initial response or the follow-on discussion, prior review of the notes is essential. The discussion should be tailored to the situation. This is not about the physician's need to impart information, and the focus should be on the needs of the relative. The start and finish of the discussion may be worrying but simple rules will make this easier. The start of the conversation must be an expression of sympathy for the loss followed by a gentle enquiry on how the family is doing and recent events. The conversation should end with an offer of future contact and an expression of support. Family preferences will dictate practicalities and the planning may cover whether the conversation is one to one or includes other members of the team or relatives. Our preference is for a focused one to one telephone conversation rather than group discussion but others may have different experiences and preferences. Lines of communication are kept open as further calls or contact may be needed as events unfold, for example, where the result of a post mortem is delayed. A face-to-face meeting with the family is offered, an offer that can be later repeated. Where relatives are well known to the team, they may find that regular contact and sharing their difficult journey is helpful. For some relatives, contact may be maintained for months or years, not only by the treating physician but also by the epilepsy nurses and secretarial staff.

The nature and content of the conversation may be recorded in the clinical record, but a letter to the GP is not our usual practice, unless specific issues arise.

c) The Face to Face Meeting 
Some families may not wish for further contact with the treating team and this must be respected. For those taking up an offer of a meeting, some may find it distressing to come to the same clinic at the treating hospital and an alternative venue may be needed. In an informal survey of 6 centres (6), all but one physician routinely offered a meeting, all but one writing immediately, with one centre opting to wait a few weeks. All stated it was unusual for the offer to be taken up. In one case a meeting with managers only was requested. In our experience, discussions are wide-ranging and may include practicalities such as the process of assessment and certification of death or tissue retention. More likely, the family will want to go over the diagnosis and medical management, the nature of any information previously given on risk of death in epilepsy, and particularly whether SUDEP could have been prevented by the family or the treating team. It is important that the bereaved are reassured that they are not to blame. Often, discussions go some way towards allaying such concerns, while at the same time acknowledging areas of uncertainty. Clarification and discussion may help mitigate against inappropriate feelings of guilt and blame.

The clinician may be concerned that an imminent initial meeting will take place in a formal setting such as at an inquest in the same court waiting room, but this should not influence the degree or the nature of contact with the family. Despite the formality, such a setting still provides an opportunity to offer condolences and sympathy to the bereaved. Inquests need not be adversarial and can be very helpful for the family especially if not unduly delayed. Going over the medical facts can help the family, and if appropriate, any shortcomings in the service can be acknowledged.

\section{Medical Record Review}

On review of medical notes, whether for a medical report, attendance at an inquest or otherwise, the following need addressing:

1. Security of diagnosis. Was there sufficient evidence on history or investigation to support the diagnosis of epilepsy, or could there have been an alternative diagnosis?

2. Documentation of the information provided by the physician or epilepsy nurses on risk, safety and self-management and specifically SUDEP (7). Such information may well have been given, but was it also recorded?

3. Assessment of ECG/cardiac risk or other relevant concomitant pathology and family history.

4. Appropriateness of the treatments offered, whether medical or surgical. 
5. Frequency of regular review as indicated by the severity of the epilepsy and treatment options available, as well as a prompt response to the patient's concerns.

The above is usually addressed as part of routine clinical care. A systematic approach during life with appropriate audits or case reviews may be helpful in optimising care and documentation thereof and in avoiding situations where practice appears to fall short of the ideal after the event.

\section{What's in a name?}

Undoubtedly, discussions are helped if the patient and family have previously been aware of risks associated with seizures and epilepsy. SUDEP is included in information provided by our nurses and in general information packs at diagnosis. For many years, it has been our practice to inform that seizures carry a risk to life, not only through injury but also, where severe, by putting a strain on vital respiratory and cardiac function. In imparting the risk of death in a seizure, which as evidence suggests accounts for the majority of SUDEP cases, less emphasis is placed on the acronym SUDEP. This is not enough. The term SUDEP is now so well known that relatives who have not previously come across it, even if they have been informed that a patient with epilepsy may die in a seizure, may be distressed when they come across the entity SUDEP for the first time after the death. More broadly, if risks were not discussed during life, the bereaved may feel that knowing about the associated risk could have influenced choices made by the individual with epilepsy or their family that could have influenced outcome. The Fatal Accident Enquiry into two SUDEP deaths in Scotland specifically advised that "The vast majority of patients with epilepsy, or their parents or carers where appropriate, should be advised of the risk of SUDEP on first diagnosis or if, in the particular circumstances of that patient, there are exceptional circumstances for delaying immediate provision of the information, then within a very short time thereafter. Advice about the risk of SUDEP should only be withheld if there is assessed to be, in the case of a particular patient, a risk of serious harm to the patient in providing the information or the patient has learning difficulties." (8)

The clinician and voluntary organisations: In the UK, there are a number of voluntary organisations who can provide excellent support for the bereaved. SUDEP Action, formerly Epilepsy Bereaved, is a specialist charitable organisation in the UK which, founded in 1995, which provides information, support and resources to bereaved relatives as well as professionals. The service (sudep.org; support direct line: 01235 772852) developed in response to the findings of research with bereaved families by Kennelly and Riesel (2002) (9). The charity also runs an epilepsy death register to facilitate supported involvement for bereaved families wishing to contribute to research. SUDEP Action staff are very experienced. In 2016, for example, 1032, including 83 very newly bereaved, made contact with the charity. In the experience of the charity, relatives usually seek to understand rather than blame and few follow an adversarial route. When they do the charity has developed contacts with mediation services and has many examples of working with all agencies after a death to restore good relationships and facilitate good outcomes. 


\section{Conclusion}

Contact after SUDEP is good for families and also good for clinicians. We believe that helping families through such difficult times is an important part of the clinician's role.

Where optimal, contact with bereaved relatives can help the grieving process by providing information and allaying inappropriate guilt reactions. For clinicians, such contact is a duty and a privilege. It is unlikely to lead to bitterness or recriminations - the opposite is usually true. It can also help provide useful information and motivation to help support discussions with patients and families at the beginning of an epilepsy journey. Facing up to post-SUDEP discussions will make for a better neurologist. Discussions inform both relatives and clinicians, foster a supportive relationship and mitigate against adversity. The above should not replace professional input for the bereaved where needed.

SUDEP is now well recognised as a distinct entity. We need better-informed support for the bereaved in the aftermath of SUDEP. Undoubtedly, more research is needed to guide this. Our aspirations for the coming years also include targeted prevention strategies which focus on identification of higher risk individuals and circumstances, optimising medical care, better information provision to reduce risk through self-management and better detection and response to seizures. 
Key Points

In the event of SUDEP

- It is recommended that the team make early contact with the family by letter or by phone, depending on circumstances.

- A face to face meeting should be offered to answer any queries and allay concerns

- Referral to voluntary organisations is recommended

- Contact with the bereaved and support should be maintained for as long as is needed

We would like to thank Dr Phillip Smith and SUDEP Action for comments on the manuscript

References and suggestions for further reading*:

1. Hanna J, Black M, Sander JWS, Smithson WH, Appleton R, Brown S, Fish DR (2002) The National Sentinel Clinical Audit of Epilepsy-Related Death: Epilepsy-death in the shadows. https://sudep.org/national-audit-epilepsy-deaths-0 (https://sudep.org/national-audit-epilepsy-deaths-0)*

2. Nashef L, Sahlholdt L. Bereavement and Sudden Unexpected Death in Epilepsy (SUDEP). In: Sudden Death in Epilepsy: Forensic and Clinical Issues. Editors: C.M. Lather, Michael W. Bungo, J.E. Leetsma pp 937-942. CRC Press. Taylor and Francis Group, LLC, USA 2011

3. Bellon M, Panelli RJ, Rillotta F. Epilepsy-related deaths: An Australian survey of the experiences and needs of people bereaved by epilepsy. Seizure. 2015 Jul;29:162-8. doi: 10.1016/j.seizure.2015.05.007. Epub 2015 May 15.

4. Nashef L, Garner S, Sander JW, Fish DR, Shorvon SD. Circumstances of death in sudden death in epilepsy: interviews of bereaved relatives. J Neurol Neurosurg Psychiatry. 1998 Mar;64(3):349-52.

5. https://www.nice.org.uk/guidance/cg137/ifp/chapter/Sudden-unexpected-death-inepilepsy-SUDEP\#if-a-person-has-died-unexpectedly 
6. Leach JP. SUDEP discussions with patients and families, Practical Neurology 2012;12:103-106

7. Donner EJ, Waddell B, Osland K, Leach JP, Duncan S, Nashef L, Picot MC. After sudden unexpected death in epilepsy: Lessons learned and the road forward. Epilepsia. 2016 Jan;57 Suppl 1:46-53. doi: 10.1111/epi.13235.

8. http://www.scotland-judiciary.org.uk/RSS/Fatal-Accident-Inquiries*

9. Kennelly C, Riesel J. Sudden Death and Epilepsy. The views and experiences of bereaved relatives and carers. Report 2002. Summary. https://sudep.org/sudden-death-andepilepsy* 